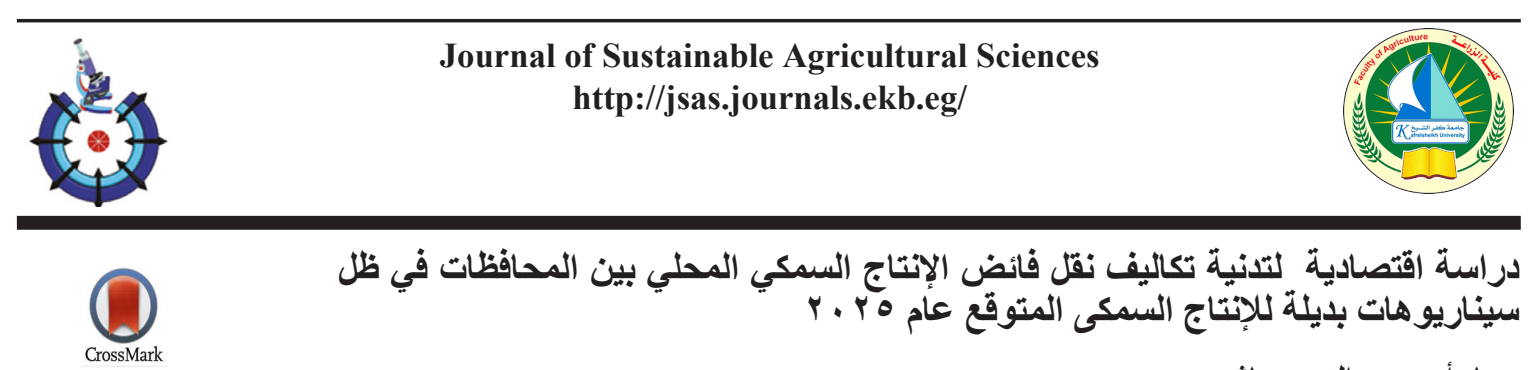

قسم العلوم الزر اعية البيئية، كلية الدراسات العليا و البحوث البيئية، جامعة عين شمس، القاهرة، مصر

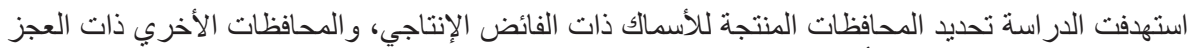

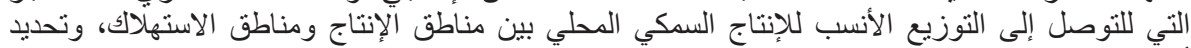

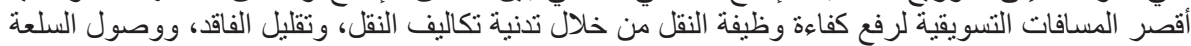

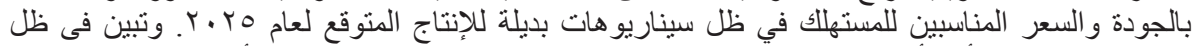

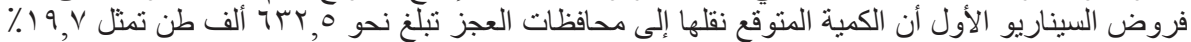

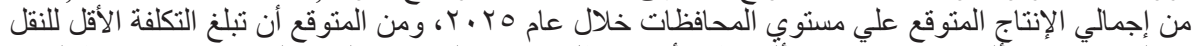

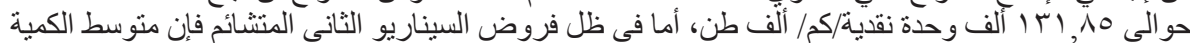

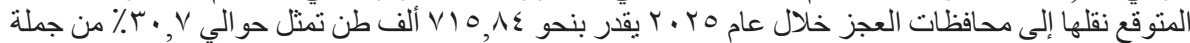

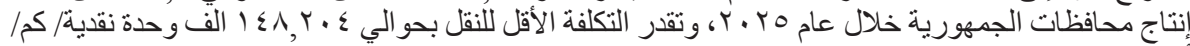

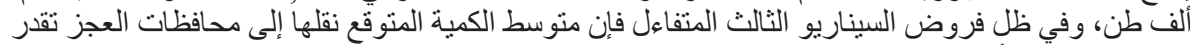

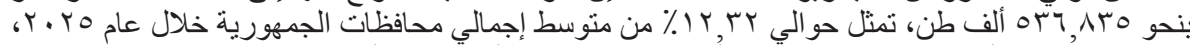

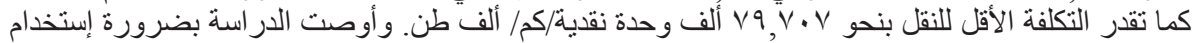

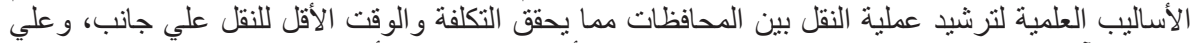

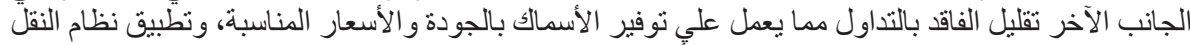

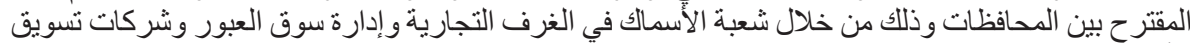

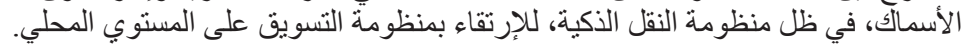

الكلمات الدالة: نموذج النقل، استهلاك الأسماك، الإنتاج المحلى من الأسماك، متوسط نصيب الإنى الفرد من من الأسماك، العجز فى الإنتاج، الفائض في الإنتاج، معدل الإنتاج النمو السكانى.

سد الفجوة بين العرض والطلب في السوق المحلى، وهو ما كانت

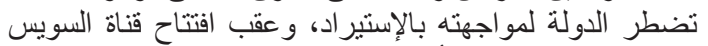

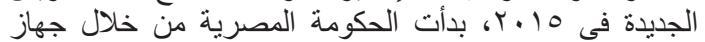

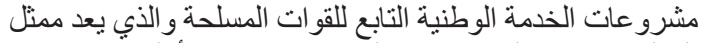

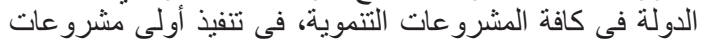

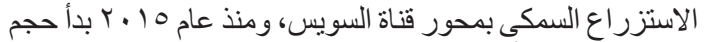

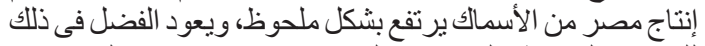
للمزارع السمكية، التى ساهم المنتج منها في زئهل فيادة إجمالي الإنتاج

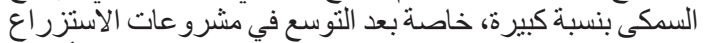

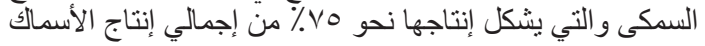
في مصر.

و هذا ما أثنتته الأرقام و البيانات الرسمية الصادرة عن الجهاز

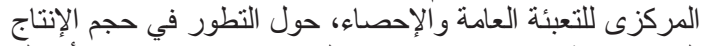

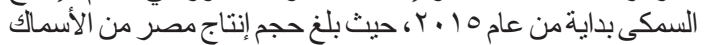

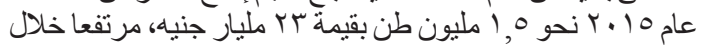

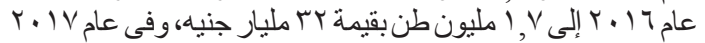

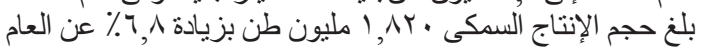

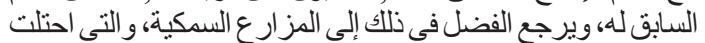

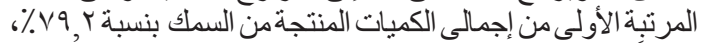

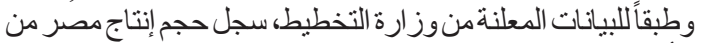

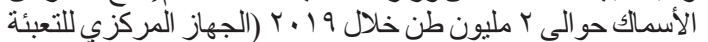

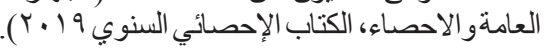

المقدمة

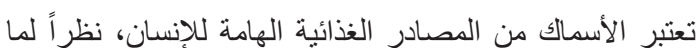

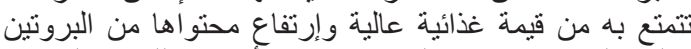

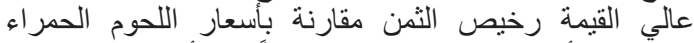

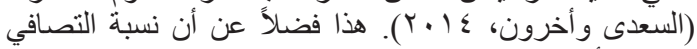

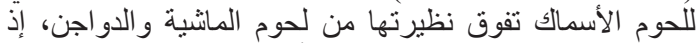

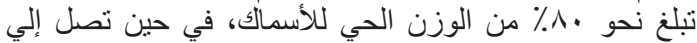

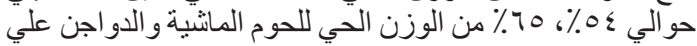

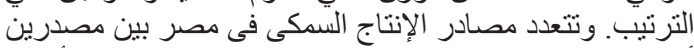

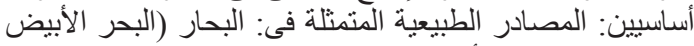

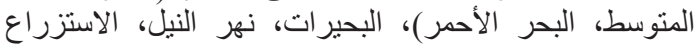

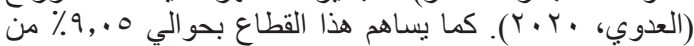

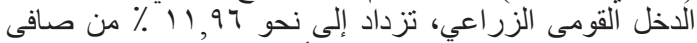

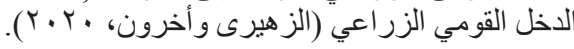

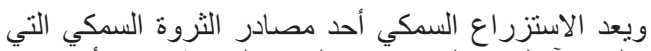

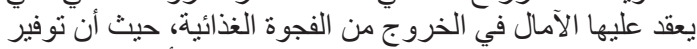

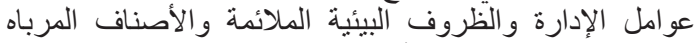

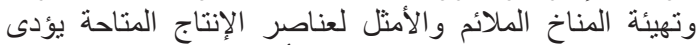

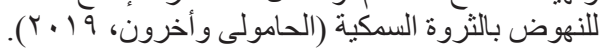

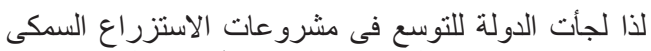

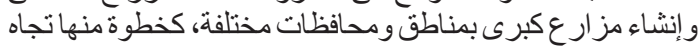


مشكلة برمجة خطية خاصة تنشأ في العديد من التطبيقات العملية

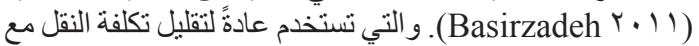

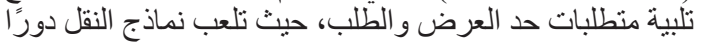

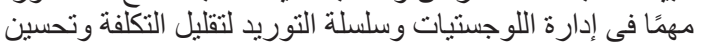

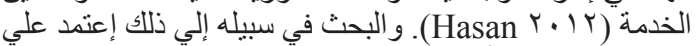

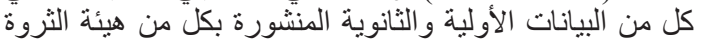

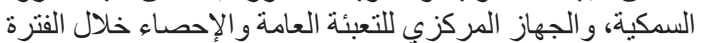

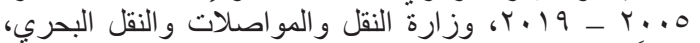
أيضاً الأبحاث و الدر اسات المتعلقة بموضو النق والبوات البحث.

$$
\text { المعالجة الإحصائية للبيانات: }
$$

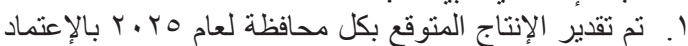

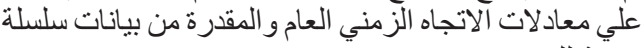

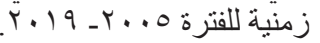

r. تم تقدير عدد السكان المتوقع' بكل محافظة بالاعتماد علي

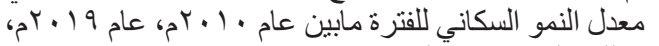
وذللك علي مستوب المحافظات.

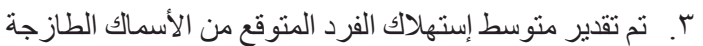

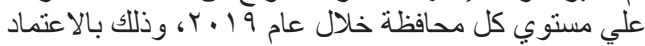

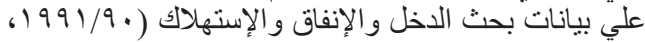

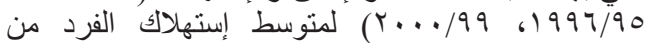

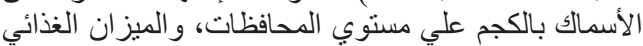

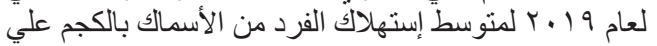

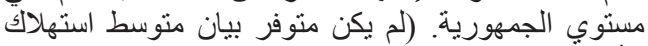
الأسمآك على مستوى المحافظات لذا تم تقديره).

ع. تم تقدير الإستهلاك المتوقع بكل محافظة خلال فترة الدراسة

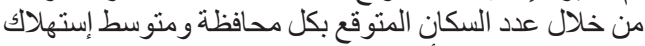
الفرد المتوقع من الأسماك بتلك الكن المحافظات.

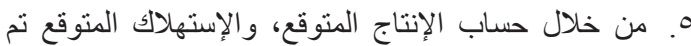

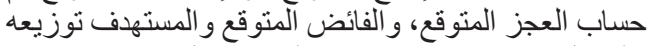

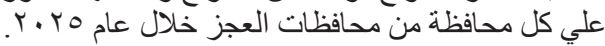

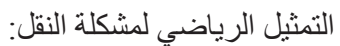

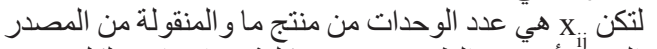

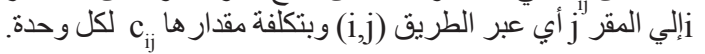

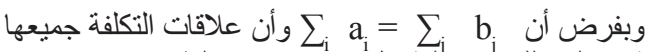
خطية، و علي ذلك فإن تكلفة نقل

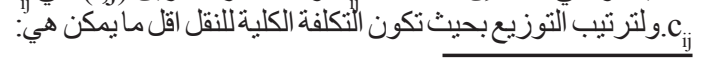

أهمية البحث أنمي

ترجع أهمية البحث إلي كونه أحد الدراسات التطبيقية التي التي التئية

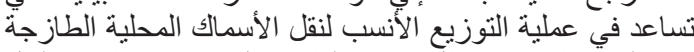

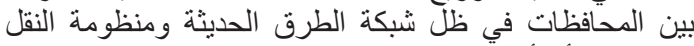
الذكية التي أنشأتها ألدولة.

مشكلة البحث

تمثل عمليات نقل الأسماك أحد الوظائف التسويقية المر احل

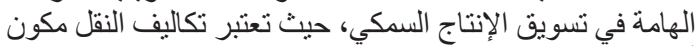

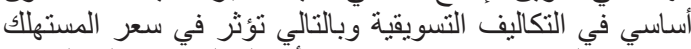

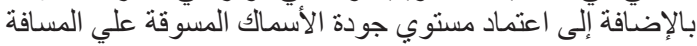

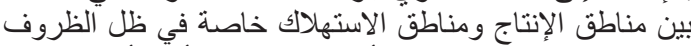

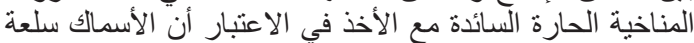

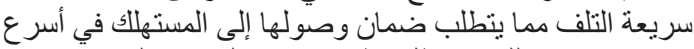

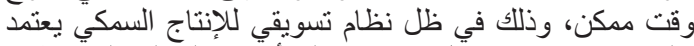

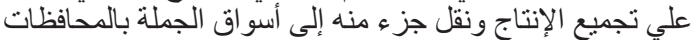

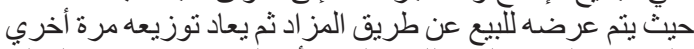

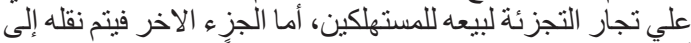

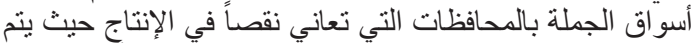

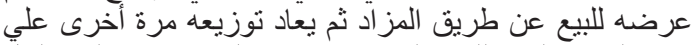

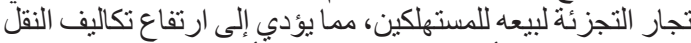
و انخفاض جودة الأسماك وبالتالي ارتفاع أسعار البيع للمستهلك. هدف البحث

تلهذف البحث إلي تحديد المحافظات المنتجة ذات الفائض

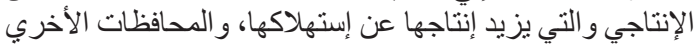

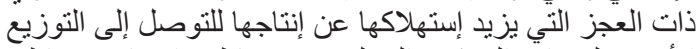

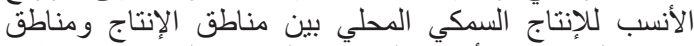

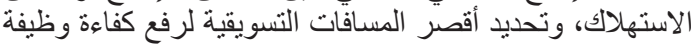

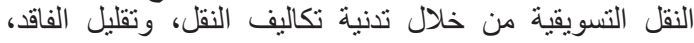
ووصول السلعة بالجودة والسعر المناسبين للمستهلكئ.

الطريقة البحثية ومصادر البيانات

إستخدم البحث أسلوب الإنحدار الخطي للتنبؤ بالإنتاج

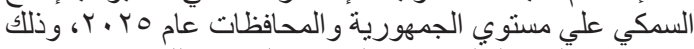

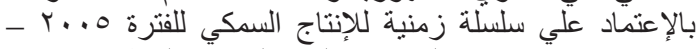

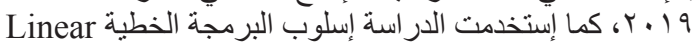

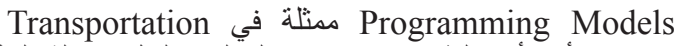
كأحد أهم الطرق في بحوث العمليات لحل مشكلة النقل Transportation Problem

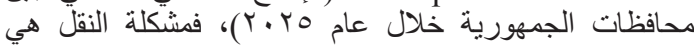

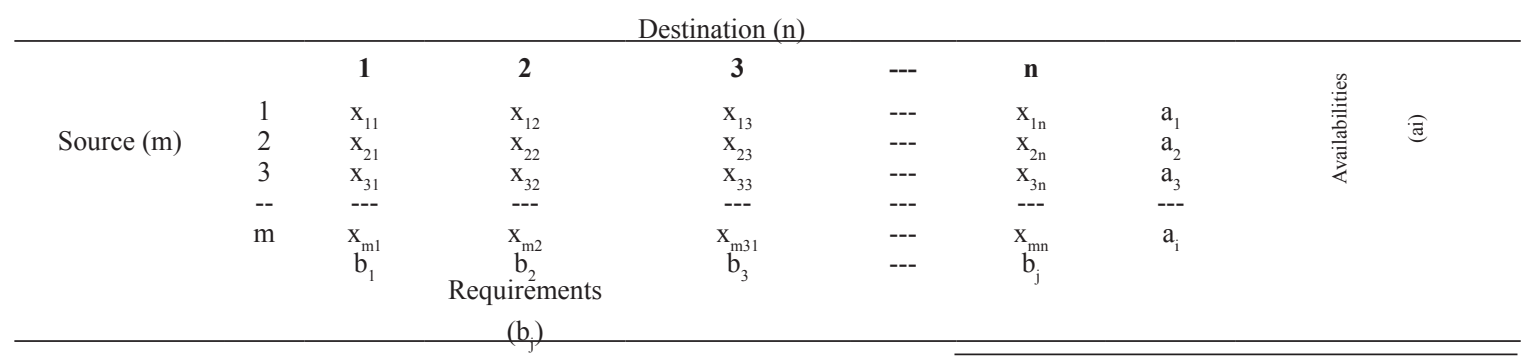

$1 \mathrm{Pt}=\mathrm{Po} \operatorname{Exp}(\mathrm{rt})$

Where: $\quad$ عt = عدد السكان المتوقع في السنة

Po = عدد السكان في سنة الأساس في السن

$$
\text { r الزمن = النمدل }
$$

$\mathrm{r}=(\ln \mathrm{pt}+1-\ln \mathrm{pt}) / \mathrm{n}$

Where: $\quad r=2$ معدل النمو السكاني

عt+1 = عدد السكان في التعداد اللاحق السكان

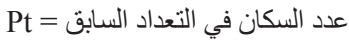


ومحافظة جنوب سيناء أنها لم تأخذ إتجاهاً عاماً معنوي

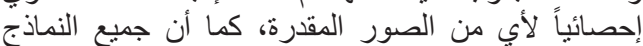

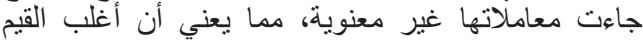
تدور حول وسطها الحسابي وذللك نتيجة لعدم استقر ار بيانت أنيات السلسلة الزمنية خلال فترة الدر اسة

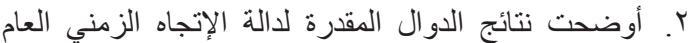

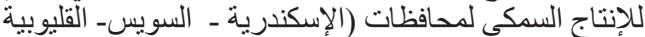

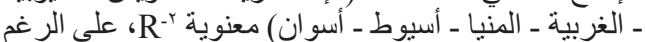

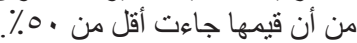

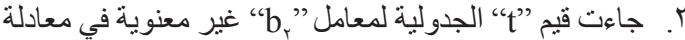
الإتجاه العام للإنتاج السمكي بمحافظة السويس. لئل

ثانياً: تقدير حدود الإنتاج السمكي المحلي المتوقع علي مستوي

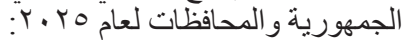

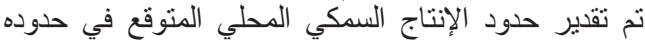

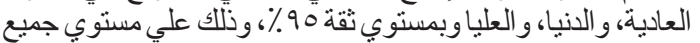

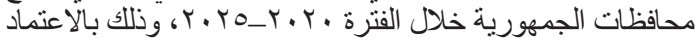

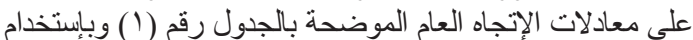

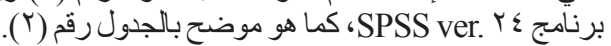
يلاحظ من الجدول رقم (r) أن جملة الإنتاج السمكي المحلي

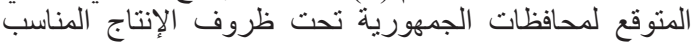

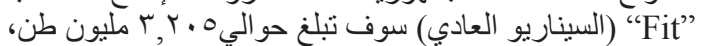

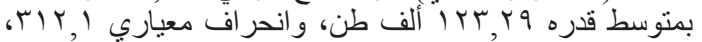

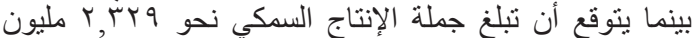

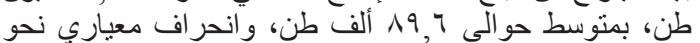

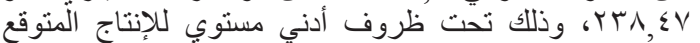

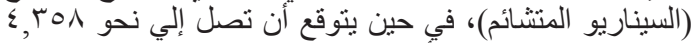

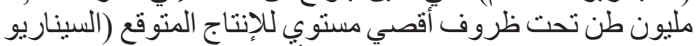

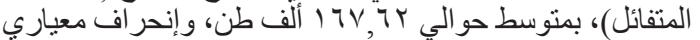
r.

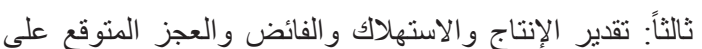
مستوي جميع محافظات الجمهورية:

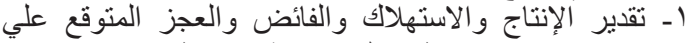

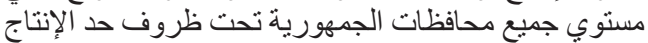

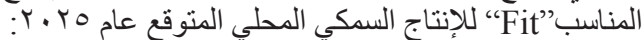

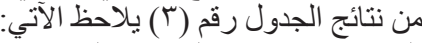

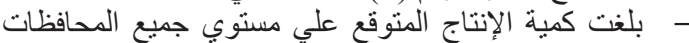

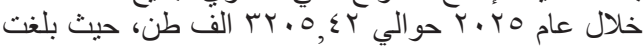

Minimize

$$
\sum_{\mathrm{i}} \sum_{\mathrm{j}} \mathrm{c}_{\mathrm{ij}} \mathrm{x}_{\mathrm{ij}}
$$

Subject to $\mathrm{x}_{\mathrm{ij}} \geq \cdot$, (One- way traffic),

and $\mathrm{m}+\mathrm{n}$ equations

in

$\mathrm{m} \times \mathrm{n}$ variables

$\sum_{\mathrm{j}} \mathrm{x}_{\mathrm{ij}}=\mathrm{a}_{\mathrm{i},}$ ( $\mathrm{m}$ supply equations)

ويكون الحل بأعداد صحيحة إذا كانت كل من a صحيحة ليست سالبة بأعل

النتائج البحثية ومناقشتهـا

أو لاً: تقدير معادلات الاتجاه الزمني العام للإنتاج السمكي المحلي

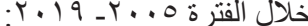

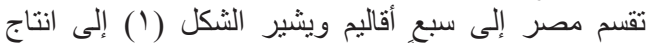

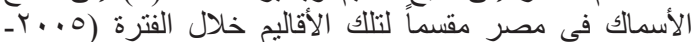

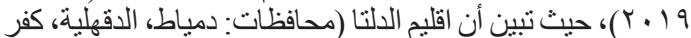

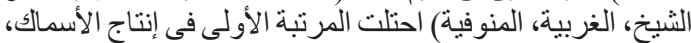

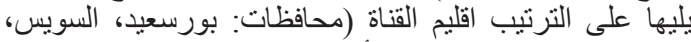

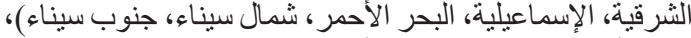

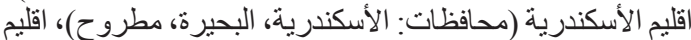

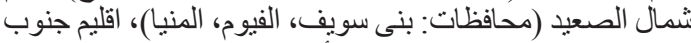

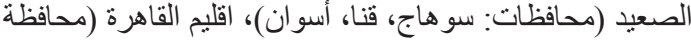
القاهرة)، اقليم اسيوط (محافظات: اسيوط، الو ادى الجدئ) الجيد).

وقد أوضحت نتائج البحث أن الإنتاج السمكي المحلي من البحني

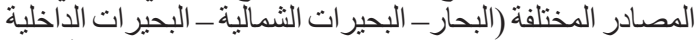

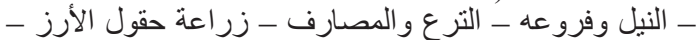

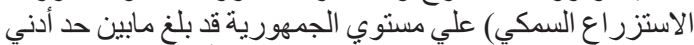

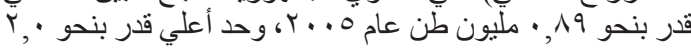

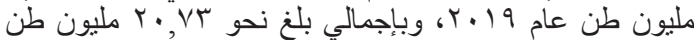

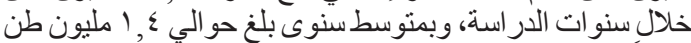

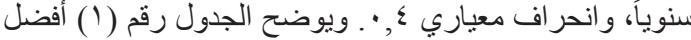

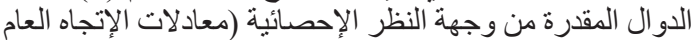

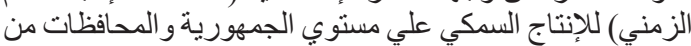

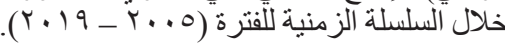
بلاحظ من الجدول رقم (1) الآتي:

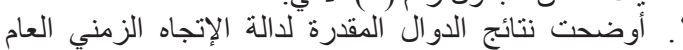

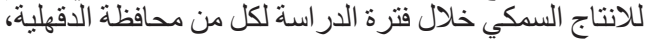

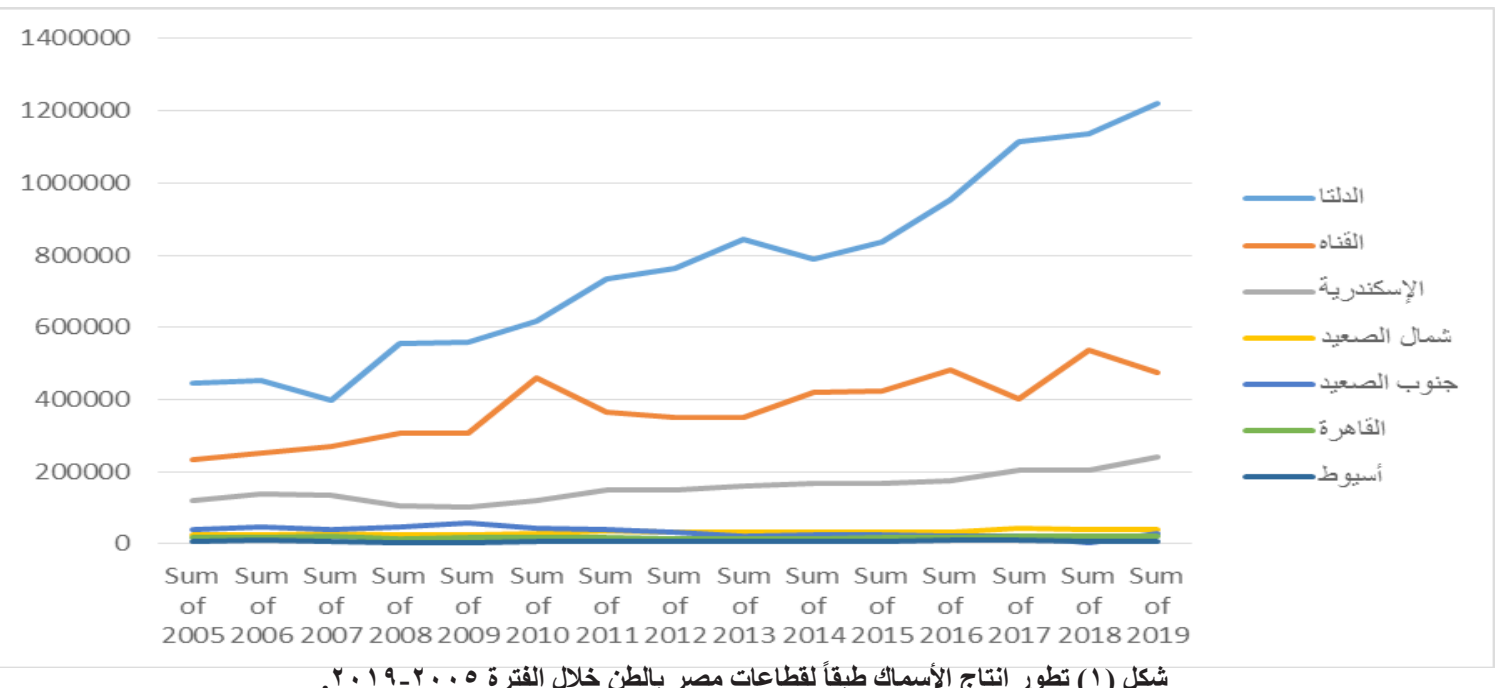

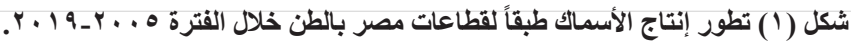




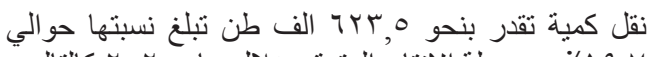

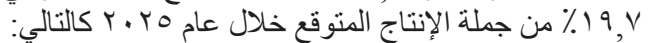

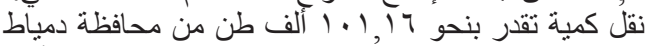

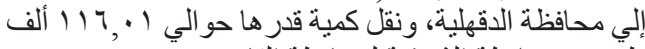
طن من محافظة الثرقية لمحافظة القاهرة.

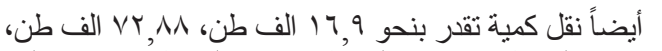

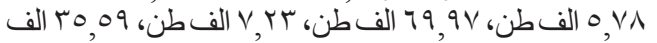
طن،

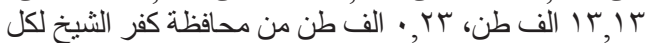

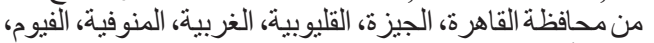
المنيا، أسيوط، سو هاج، قنا، الو ادي الجديد لكل منها علي الترتيب.

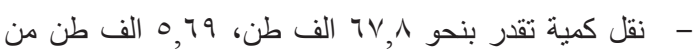

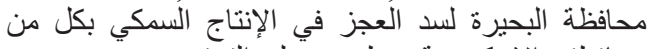
محافظتي الإسكندرية ومطروح علي الترتيب.

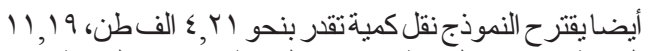

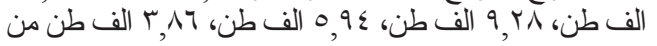

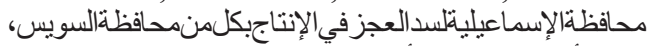

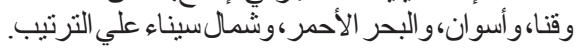

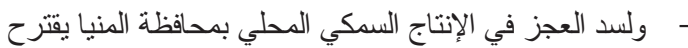

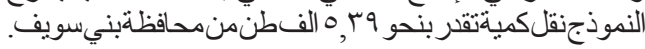

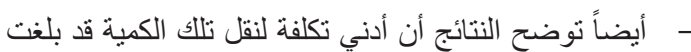

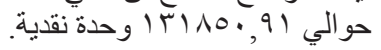

r- التوزيع الأنسب لفائض الإنتاج السمكي المحلي المتوقع بين

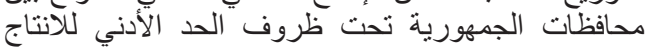

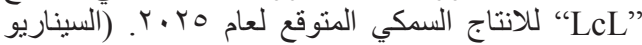

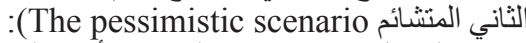
يوضح الجدول رقم (V) نتائج التوزيع الأنسب لفائض الإنتاج

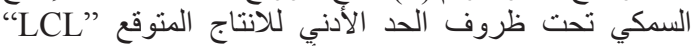

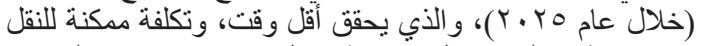

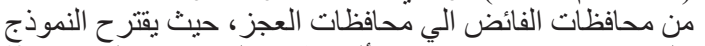

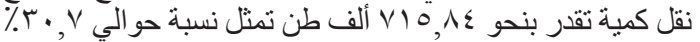

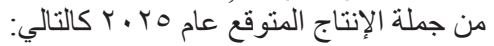
-

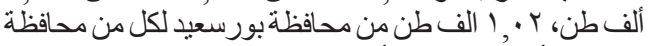
السويس، أسوان، البحر الأحمر ، شمال سيناء علي الترنيب.

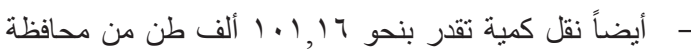

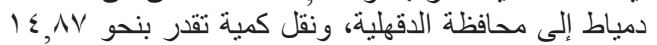
ألف طن من محافظة الثرقية لمحافظة أسو ان.

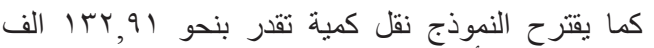

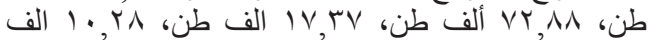

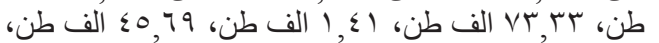

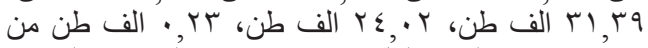

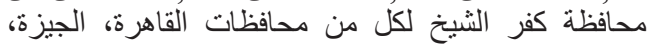

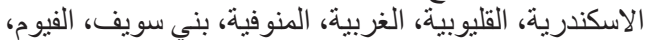

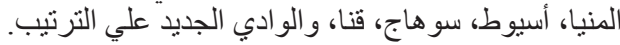

ولسد العجز بمحافظتي الأسكندرية ومطروح يقتر ح نقل كمية

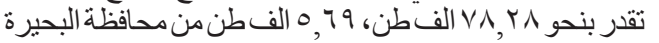

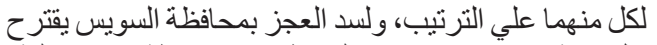

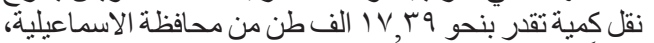
أيضاً ولسد العجز بمحافظة شمال سيناء يقتر ح نقل كمية نقدر

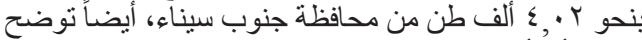

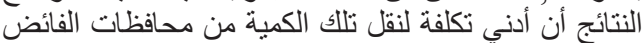

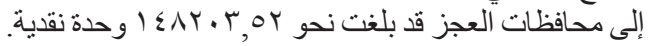

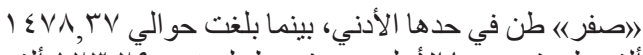

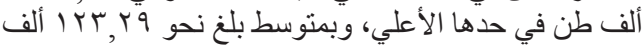

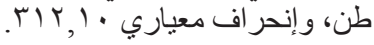

جاءت محافظة كفر الثيخ في المرنبة الأولي حيث بلغت كمية الإنية

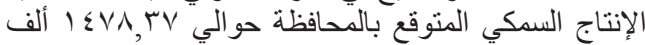

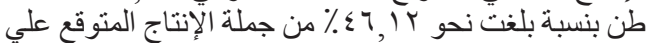

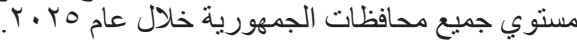

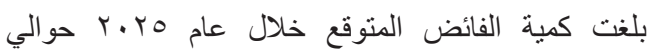

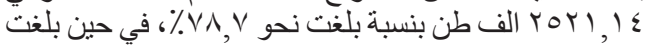

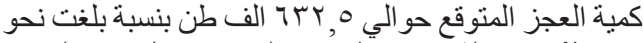

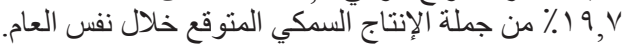

r- تقدير الإنتاج والاستهلاك و الفائض والعجز المتوقع علي

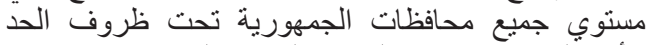

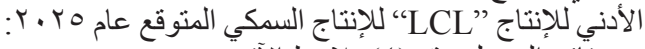

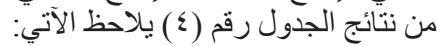

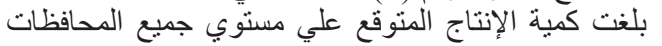

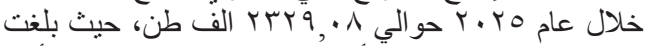

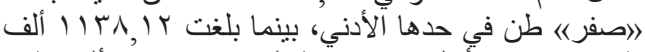

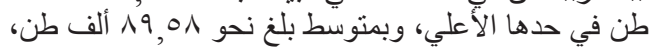

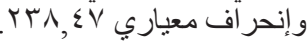

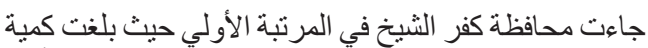

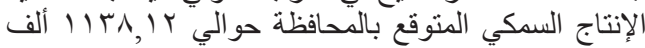

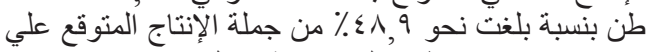

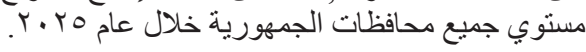

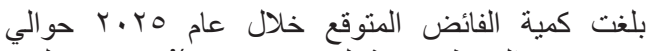

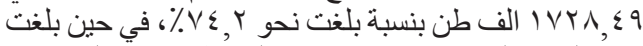

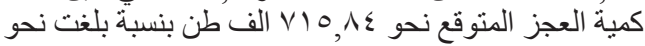

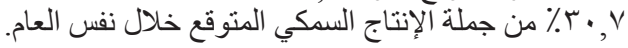

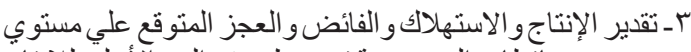
جميع محافظات الجمهورية تحت ظروف الإن الحد الأعلي للانتاج

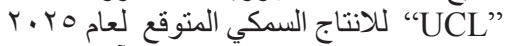
من نتائج الجدول رقم (0) يلاج الاحظ الآتي:

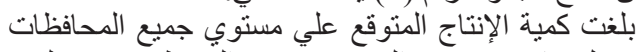

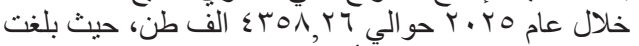

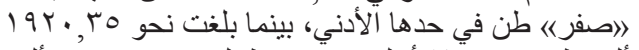

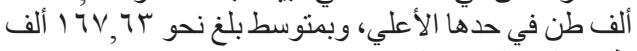

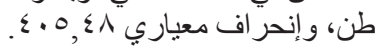

جاءت محافظة كفر الشيخ في المرتبة الأولي حيث بلغت الإنت

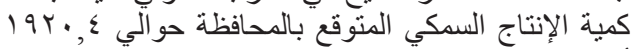

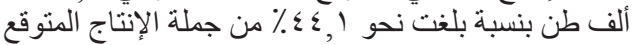

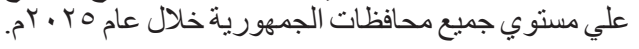

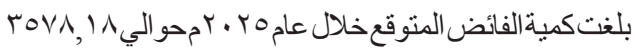

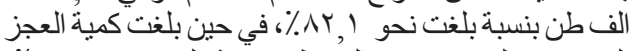

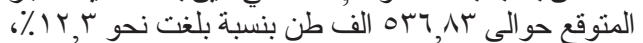
و ذللك من جملة الإنتاج السمكي المتوقع خلال نفس العام.

رابعاً: التوزيع الأنسب لفائض الإنتاج السمكي المحلي المنوقع بين

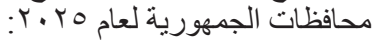
1ـ التوزيع الأنسب لفائض الإنتاج السمكي المحلي المتوقع بين

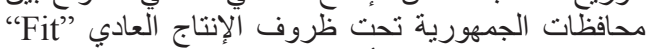

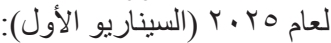

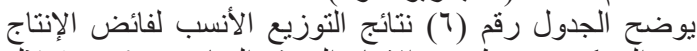

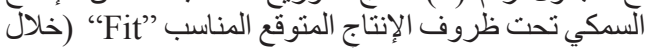

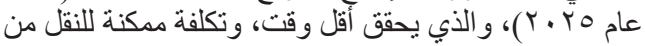

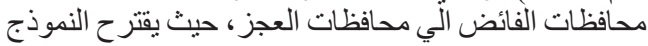

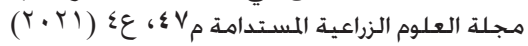




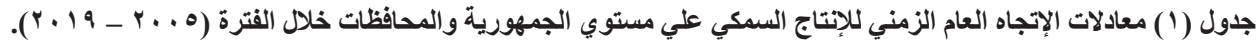

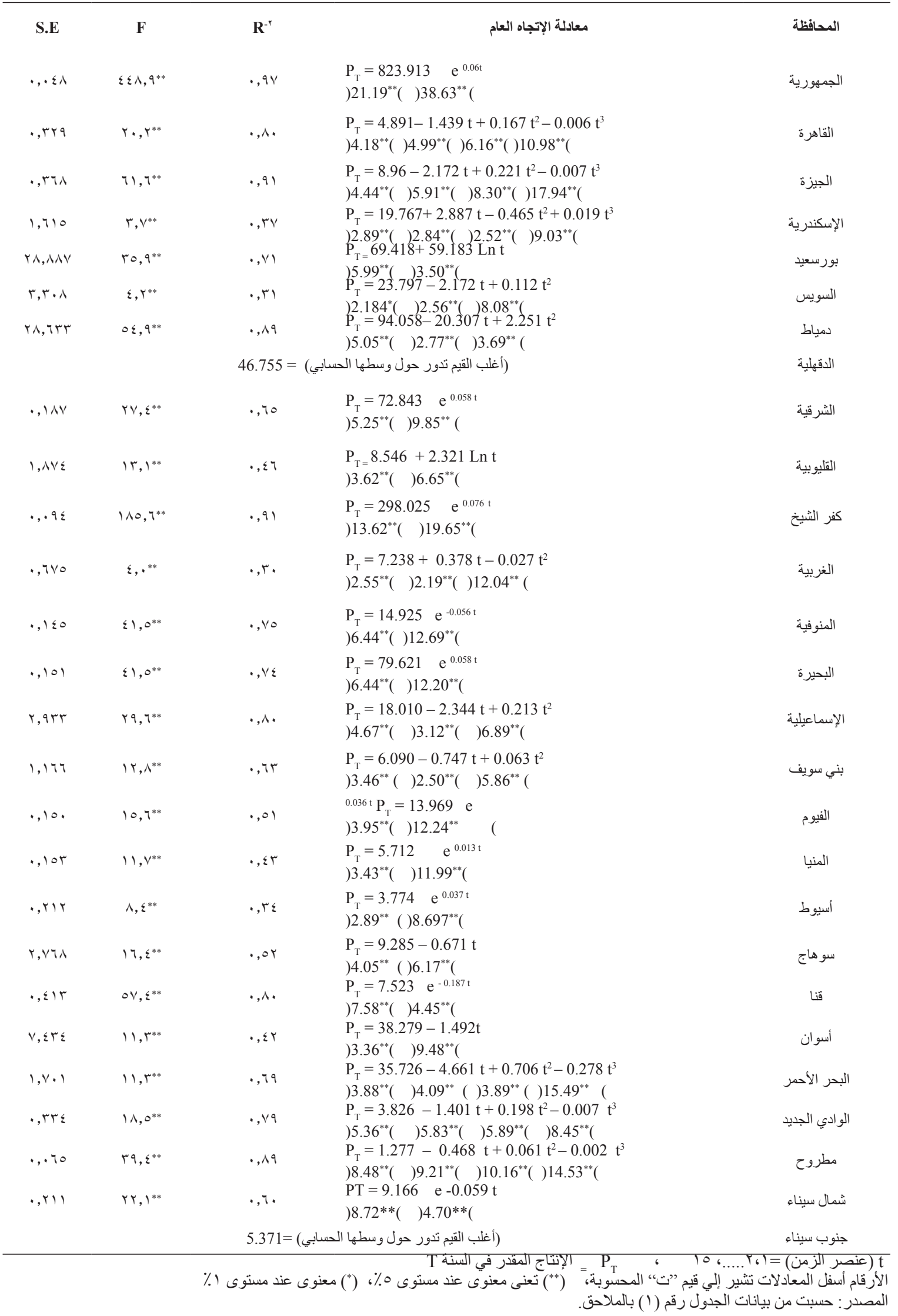

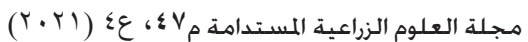




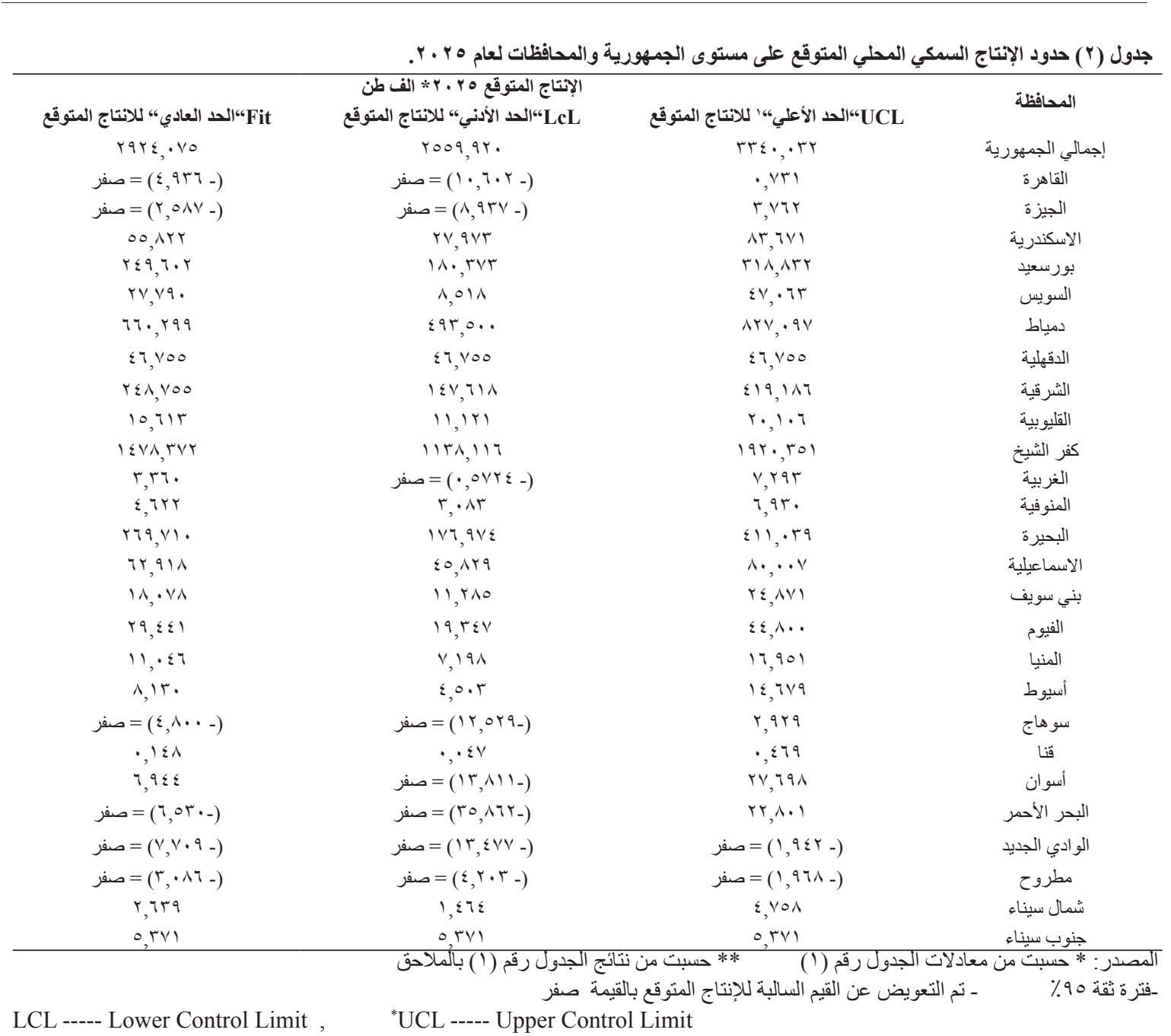

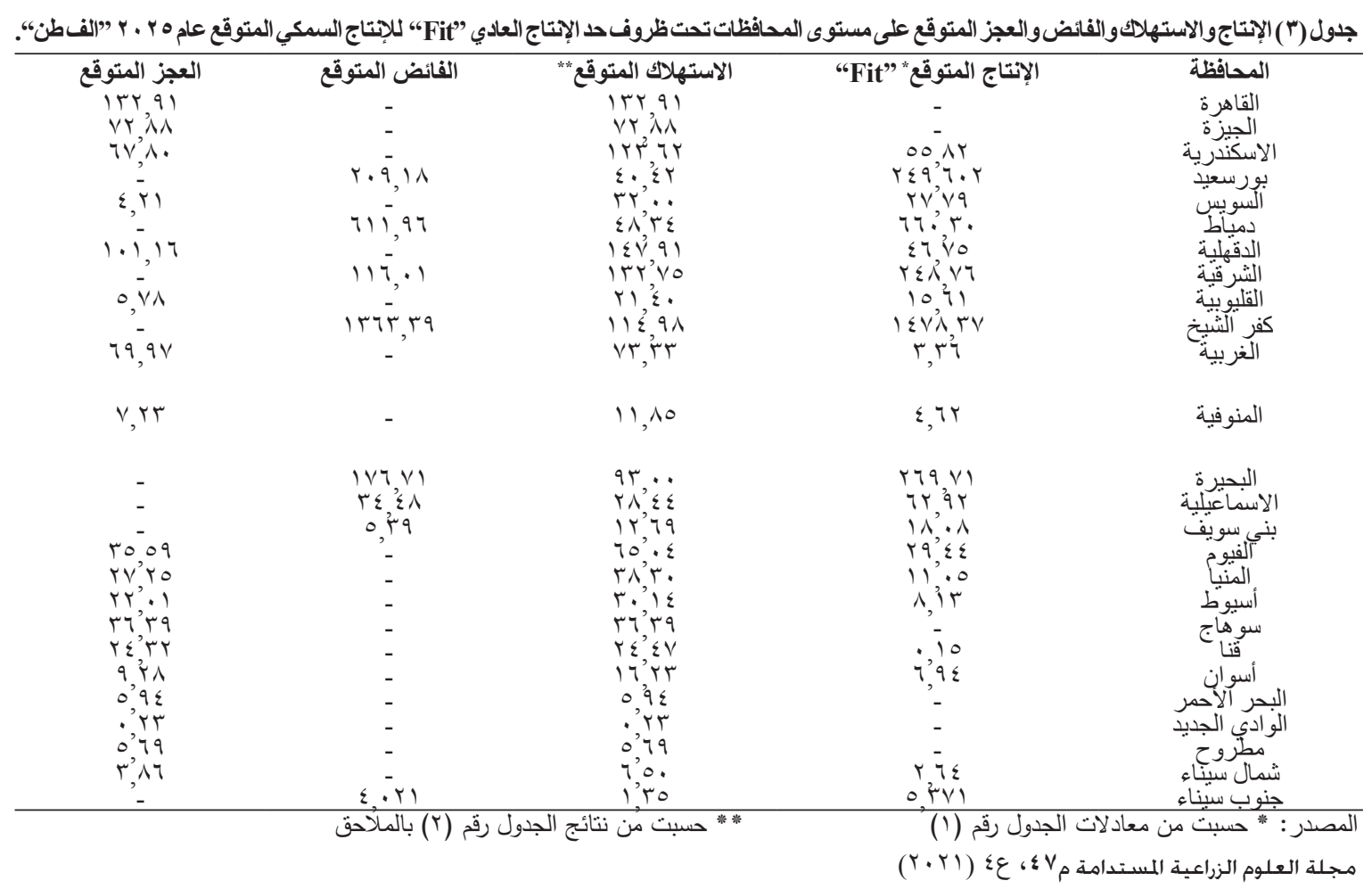




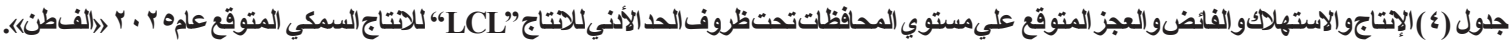

\begin{tabular}{|c|c|c|c|c|}
\hline العجز المتوقع & الفائض المتوقع & الاستهلاك المتوقع** & "الإنتاج المتوقع "LCL" & المحافظة \\
\hline$-\mid r r, q 1$ & - & $\mid r r, q 1$ & - & القاهرة \\
\hline$V Y, \wedge \wedge-$ & - & $V Y, \wedge \wedge$ & - & الجيزة \\
\hline $90,70-$ & - & ITr, Tr & rV, qVT & الاسكندرية \\
\hline- & 119,90 & $\varepsilon \cdot, \varepsilon r$ & $11 \cdot, r V T$ & بورسعيد \\
\hline$r r, \Sigma \Lambda-$ & - & $r, .$. & $\wedge, 011$ & السويس \\
\hline- & $\varepsilon \leqslant 0,10$ & $\varepsilon \wedge, \Gamma \varepsilon$ & $\varepsilon q \pi, 0 \ldots$ & دمياط \\
\hline $1.1,17=$ & - & $1 \leq v, q 1$ & $\leqslant 7,100$ & الدقهلية \\
\hline- & $1 \leq, \wedge V$ & Ir, Vo & $1 \leq V, 71 \wedge$ & الشرقية \\
\hline $1 \cdot, r \wedge-$ & - & $Y_{1}, \varepsilon$. & $11,|Y|$ & القليوبية \\
\hline- & $1 \cdot r r, 1 \leq$ & $11 \leqslant, 91$ & $11 \% \wedge, 117$ & كفر الثيخ \\
\hline _ שr,T & - & מז, & - & الغربية \\
\hline$\wedge, \vee V-$ & - & 11,10 & $r, \cdot r$ & المنوفية \\
\hline - & Nr, qv & $94, \ldots$ & $1 \vee 7,9 \vee \varepsilon$ & البحيرة \\
\hline- & IV,rq & $r \wedge, \varepsilon \varepsilon$ & $\varepsilon 0, \wedge r q$ & الاسماعيلية \\
\hline $1,\{1-$ & - & 15,79 & $11, r \wedge 0$ & بني سويف \\
\hline$\leqslant 0,79$. & - & $10, \cdot \varepsilon$ & $19, r \leq V$ & الفيوم \\
\hline $1,1=$ & - & rᄉ,r. & $v, 191$ & المنيا \\
\hline Yo,' $7 \leqslant-$ & - & 5 & $\varepsilon, 0 . r$ & أسيوط" \\
\hline - & - & rq, & - & سو هاج \\
\hline$r \varepsilon, r-$ & - & $r \leqslant, \leqslant V$ & $\cdot, \leqslant 77$ & قنا \\
\hline $17, r{ }_{-}$ & - & $17, r T$ & - & أسوان \\
\hline $0,9 \leq-$ & - & $0,9 \leq$ & - & البحر الأحمر \\
\hline$\cdot, r{ }_{-}$ & - & •, & - & الو ادي الجديد \\
\hline $0,79-$ & - & 0,79 & - & مطروح \\
\hline $0, \cdot \varepsilon-$ & - & $7,0$. & $1, \varepsilon \div \varepsilon$ & شمال سيناء \\
\hline- & $\varepsilon, r$ & ס 1,1 & $0, r v i$ & جنوب سيناء \\
\hline
\end{tabular}

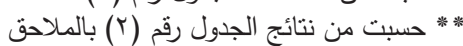

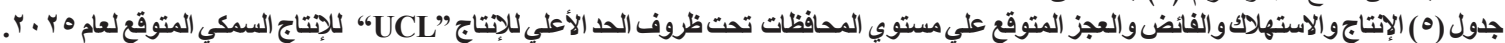

\begin{tabular}{|c|c|c|c|c|}
\hline العجز المتوقع & الفائض المتوقع & الاستهلاك المتوقع** & "الإنتاج المتوقِق "UCL" & المحافظة \\
\hline Irr, IN- & - & $\mid r r, q 1$ & $\cdot, V T$, & القاهرة \\
\hline $79, i r_{-}$ & - & $V Y, \wedge \Lambda$ & rotr & الجيزة \\
\hline$r q, 90_{-}$ & - & TrT, & $\Delta r, T \times 1$ & الاسكندرية \\
\hline- & $r \vee \wedge, \varepsilon 1$ & $\varepsilon \cdot, \leqslant r$ & rו^,גז & بورسعيد \\
\hline- & $10, \cdot 7$ & $r r, \ldots$ & $\varepsilon V, \cdot T r$ & السويس \\
\hline- & $V \vee A, V T$ & $\varepsilon \Lambda, r \leqslant$ & ArV, 9V & دمياط \\
\hline $1.1,17$ & - & $1 \leqslant v, q 1$ & $\leq 7, \vee 00$ & الدقهلية \\
\hline- & KAт, $\leqslant \varepsilon$ & Irt, vo & $\{19,117$ & الشرقية \\
\hline $1, q_{-}$ & - & $r_{1}, \varepsilon$. & $r \cdot, 1 \cdot r$ & القليوبية \\
\hline- & $11.0, r V$ & $11 \leqslant, 91$ & $194 \cdot, r 01$ & كفر الثيخ \\
\hline $77, \cdot \varepsilon_{-}$ & - & שr, & v,rar & الغربية \\
\hline$\varepsilon, q r_{-}$ & - & 11,10 & 7', 94. & المنوفية \\
\hline- & Mハ, $: \varepsilon$ & $q 4, .$. & $\leqslant 11,49$ & البحيرة \\
\hline- & $01,0 \mathrm{~V}$ & $r \wedge, \varepsilon \varepsilon$ & $\wedge,, \cdots \vee$ & الاسماعيلية \\
\hline- & $|r| 1$, & $1 r, 79$ & $r \leqslant, \wedge Y \mid$ & بني سويف \\
\hline$r \cdot, r \varepsilon_{-}$ & - & $70, \cdot \varepsilon$ & $\varepsilon \varepsilon, \wedge \ldots$ & "الفيوم \\
\hline ri, & - & ॅ^,ॅ. & 17,901 & المنيا \\
\hline $10, \leq 7$ & - & $r \cdot, 1 \leq$ & $1 \leq 7 \vee 9$ & أسيوط \\
\hline rr', & - & q & r,qrq & سوهاج \\
\hline$r \leqslant, \cdot-$ & - & $r \leqslant, \leqslant V$ & $\cdot, \leqslant 79$ & قنا \\
\hline- & $11, \leqslant V$ & $17, r T$ & $r v, 791$ & أسوان \\
\hline- & $17, \wedge 7$ & $0,9 \varepsilon$ & $r, \wedge, 1$ & البحر الأحمر \\
\hline$\cdot, Y^{T}$ & - & • & 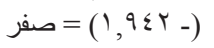 & الو ادي الجديد \\
\hline 0,79 & - & 0,79 & ( & مطروح \\
\hline $1, V \varepsilon_{-}$ & - & $7,0$. & $\varepsilon, \vee \circ \wedge$ & شمال سيناء \\
\hline- & $\varepsilon, \cdot r$ & $1, r_{0}$ & $0, r_{1}$ & جنوب سيناء \\
\hline
\end{tabular}

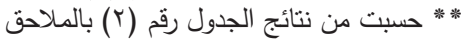

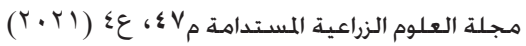




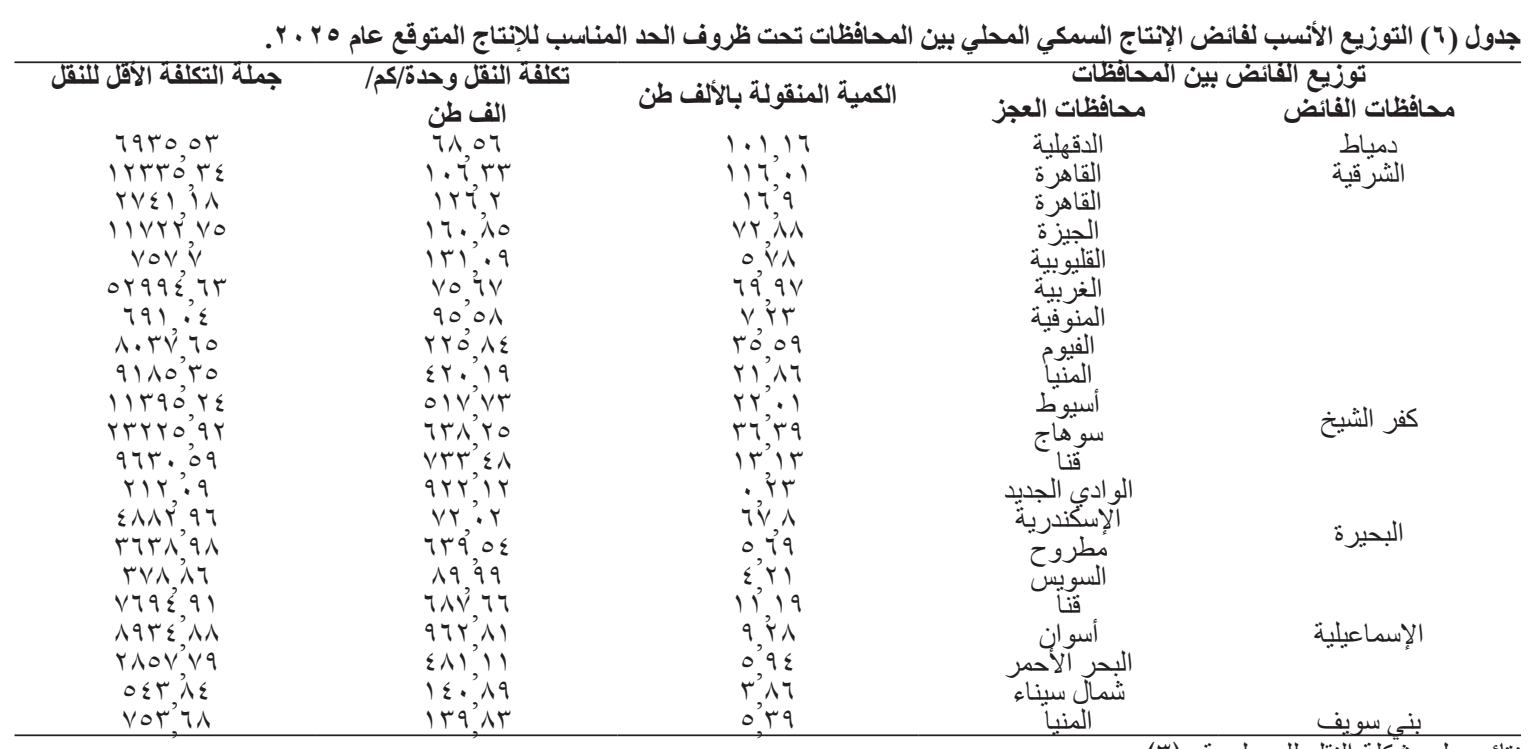

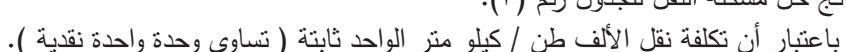

\begin{tabular}{|c|c|c|c|c|}
\hline \multirow[t]{2}{*}{ جملة التكلفة الاقلى للنقفل } & \multirow{2}{*}{ تكلفة النقل وحدة"/كم/ } & \multirow{2}{*}{ الكمية المنقولة بالألف طن } & \multicolumn{2}{|c|}{ توزيع الفائض بين المحافظات } \\
\hline & & & محافظات العجز & محافظات الفائض \\
\hline $\begin{array}{l}1 \cdot r \cdot, \leqslant q \\
1 \leqslant \cdot v i\end{array}$ & $\begin{array}{l}179,51 \\
1.42 \% 7\end{array}$ & 79י9יר & ألسوبس أبس & \\
\hline$r r \cdot \Lambda, q \varepsilon$ & oov,, 1 & $0,9 \leq$ & & بورسعيد \\
\hline KYY, To & YIV, & $I^{\prime}, Y$ & شمال سيناء & \\
\hline $7970^{\circ}$ or & 40,04 & $1,1,17$ & الدقاقهية & دياط. \\
\hline $1 \leq \varepsilon 0 \hat{v}$ & $9 Y Y, Y A$ & I ¿Av & 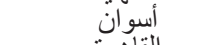 & الثرقية \\
\hline $111+i r$ & צ' & $\mid r \%, 91$ & القاهرة - ماهة & \\
\hline qArr, qV & $1 \% \xi 95$ & lis & 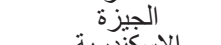 & \\
\hline 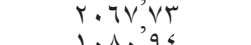 & $119,: \varepsilon$ & ive & الاسكندرية & \\
\hline 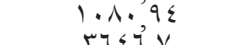 & $\begin{array}{ll}1.0,10 \\
<9,10\end{array}$ & 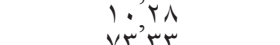 & القلبو بية & \\
\hline 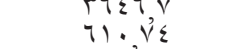 & $\begin{array}{l}\varepsilon 9,7 \overline{ } \\
79,7 \varepsilon\end{array}$ & 人, iv & اللغربية & \\
\hline 47,1 & roo, rq & $i, \leqslant 1$ & بني سويقِف & كفر الشيخ \\
\hline 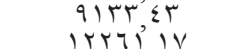 & $\begin{array}{l}1999 \\
r 9 \leq 2 \%\end{array}$ & $\{0,79$ & ألفيو & \\
\hline MY.9, vo & $\leqslant 9 i_{2} \wedge$ & ro, $7 \varepsilon$ & أسيوط & \\
\hline YYYA1'94 & 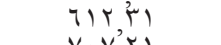 & MY, & سوضهاج & \\
\hline $179 \wedge v^{\prime}, 19$ & $V \cdot V^{\prime}, Y_{1}$ & $r \varepsilon, \cdot r$ & قَتنا & \\
\hline (15,9 & $9 K Y^{2}, y$ & rr & الو الادي الجديد & \\
\hline 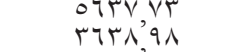 & $\begin{array}{l}V T \\
T r{ }^{\prime}\end{array}$ & $\begin{array}{l}V A, Y A \\
0,79\end{array}$ & |لمطروحرية & 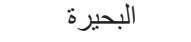 \\
\hline $107 \varepsilon, 94$ & $19,99^{\circ}$ & $1 v^{2}, \mathrm{rq}^{2}$ & السويس & الاسماعيلية \\
\hline 1.0..1 & r..., ro & $\varepsilon \cdot r$ & شمال سبناء & جنوب س \\
\hline
\end{tabular}

طن من محافظة بني سويف، ولسد العجز بمحافظة الدقهلية

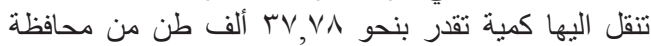
دمياط.

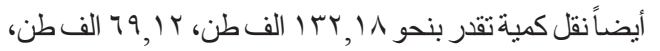

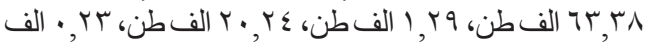

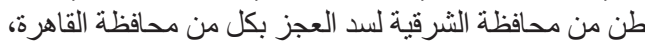
الجيزة، و الدقهلية، القليوبية، الفيوم، الو ادي الجديد علي التئ الترتيب.

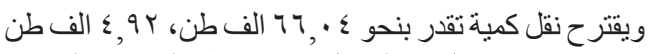

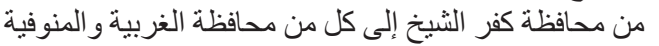

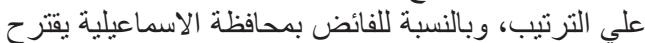

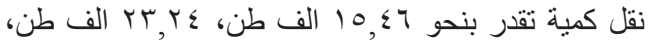
1,Vع الف طن لكل من محافظة أسيوط وسوهاج وشمال سيناء علي الترتيب. rـ التوزيع الأنسب لفائض الإنتاج السمكي المحلي المتوقع بين

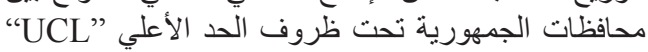

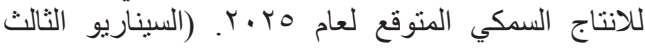

المتفائل الج - - يوضح الجدول رقم (^) نتائج التوزيع الأنسب لفائض الإنتاج

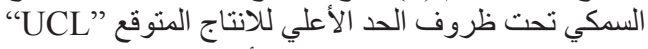

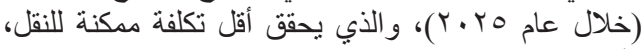

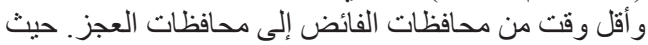

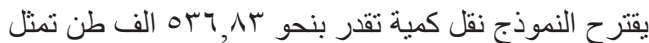

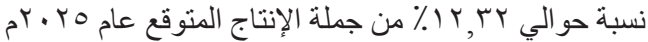
علي أن يكون توزيع نقلها كالتالي:

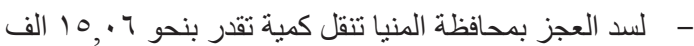

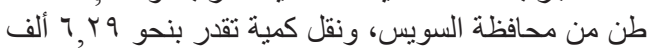

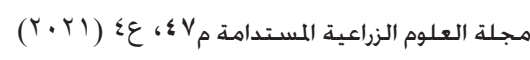




\section{التوصيات}

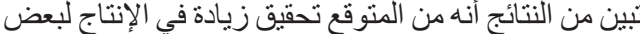

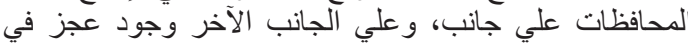

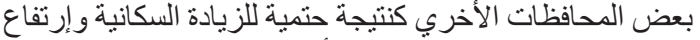

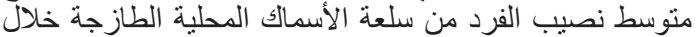

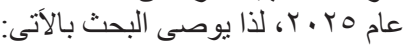

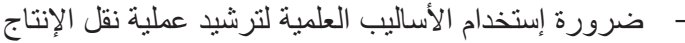

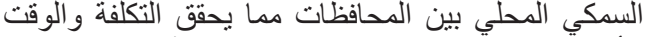

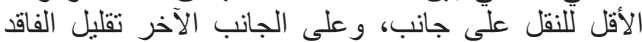

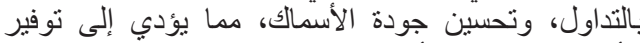
الأسماك بالجودة و الأسعار المناسبة.

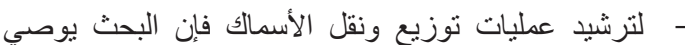

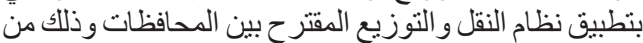

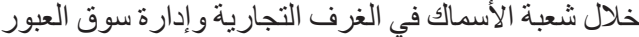

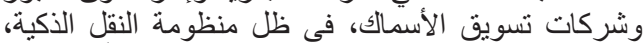
وتطور شبكة الطرق التي أنشأتها الدولة مؤخراً للإرتقاء الأنة بمنظومة النقل كوظيفة تسويقية علي المستوي المحلي.
- - ويستكمل باقي العجز في الإنتاج لمحافظة سوهاج من كل

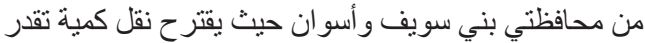
بنحو 0,09 الف طن، بr, بـ الف طن من كل من المحافظتين

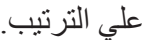

كما يقتر حسد العجز في الانتاح بمحافظة قنا بنقل كمية تقدر

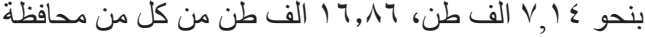
أسوان والبحر الأحمر علي الترتيب. - _ _كماً توضح النتائج أن أدني تكلفة لنقل تلك الكمية من

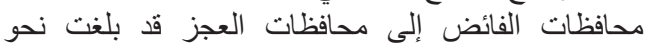

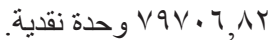

من كل ماسبق يتضح توقع تركز إنتاج الأسماك في كل من الإن محافظات كفر الثيخ، بورسعيد، دمياط، البحيرة، الاسماعيلية، الإنية،

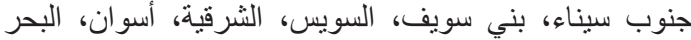

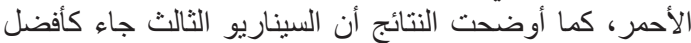

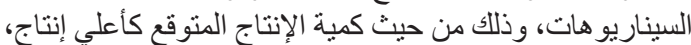

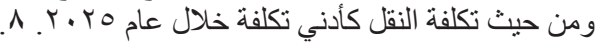

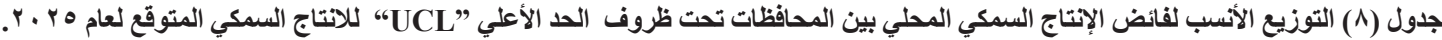

\begin{tabular}{|c|c|c|c|c|}
\hline \multirow{2}{*}{ جملة التكلفة الأقل للنقل } & \multirow{2}{*}{ تكلفة النقل وحدة/كم/ } & \multirow{2}{*}{ الكمية المنقولة بالألف طن } & \multicolumn{2}{|c|}{ توزيع الفائض بين المحافظات } \\
\hline & & & محافظات العجز & محافظات الفائض \\
\hline 0407,99 & $r 00, \times 1$ & 10,47 & 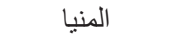 & 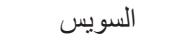 \\
\hline$r \circ 9 \cdot, r$ & 71,07 & $r v, Y \wedge$ & الدقهلية & 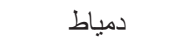 \\
\hline $1 \leqslant .0 \leqslant, V$ & 1.7, & $1 \pi, 11$ & 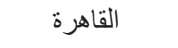 & \multirow{6}{*}{ الشرقية } \\
\hline$\vee 9 \vee 9, r)$ & $110, \leqslant \leqslant$ & $79,1 r$ & 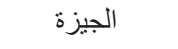 & \\
\hline$\varepsilon \cdot \varepsilon \vee, \leqslant 0$ & rT,人 & גו גזי & الدقهلية & \\
\hline $111, \pi 1$ & $\wedge \uparrow, r q$ & $1, r 9$ & القليوبية & \\
\hline$r+1, r$ & IVV,qT & $r \cdot, r \varepsilon$ & 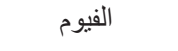 & \\
\hline$r \cdot 1, \cdot V$ & $\Delta V \leqslant, Y)$ & $\cdot, r \mu$ & الو ادي الجديد & \\
\hline TrAs, IV & $\varepsilon q, \vee r$ & $7, \cdot \varepsilon$ & 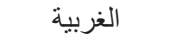 & \multirow{2}{*}{ كفر اشيخ } \\
\hline$r \leqslant r, T r$ & $79,7 \leq$ & $\varepsilon, 94$ & المنوفية & \\
\hline YAVV,, & $V Y, \cdot r$ & $r 9,90$ & الاسكندرية & \multirow{2}{*}{ 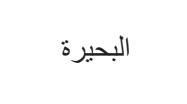 } \\
\hline 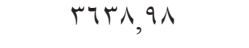 & $7 \pi 9,0 \leq$ & 0,79 & 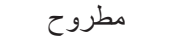 & \\
\hline$V Y 90, Y r$ & $\leq V 1,91$ & $10, \leqslant 7$ & أسيوط & \multirow{3}{*}{ الاسماعيلية } \\
\hline IrVฯA, $\cdot V$ & $09 Y, \leqslant T$ & $r T, Y \leq$ & سو هاج & \\
\hline$r \leqslant 0,10$ & $1 \varepsilon \cdot, \wedge 9$ & $1,1<\varepsilon$ & شمال سيناء & \\
\hline AVq,OT & $1 \% q, \wedge r$ & $7, Y 9$ & 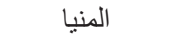 & \multirow{2}{*}{ بني سويف } \\
\hline$r 1 \cdot v, 9 v$ & $r \circ v, \wedge 9$ & $0, \wedge 9$ & سو هاج & \\
\hline $1749,9 \leq$ & $r \vee \wedge, V \varepsilon$ & $\varepsilon, \Gamma$ & سو هاج & \multirow{2}{*}{ أسوان } \\
\hline $19 \times 9,07$ & YVY, YO & $v, 1 \leq$ & قنا & \\
\hline$r v \cdot 0,7 r$ & $r 19,19$ & $17, \wedge 7$ & قنا & البحر الأحمر \\
\hline
\end{tabular}

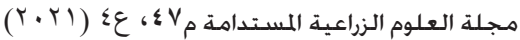


السعدى، أحمد بدير أحمد، القبلاوى، مصطفى عبد ربه محمد،

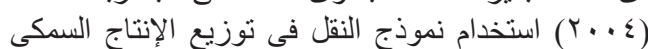

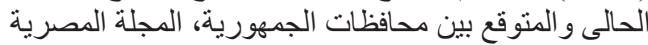

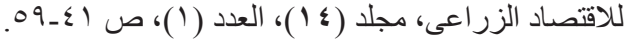

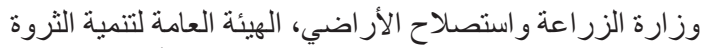

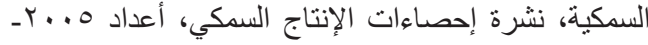

$r+19$

وزارة النقل والمواصلات و النقل البحري، الهيئة العامة للطرق إلبات

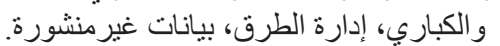

Basirzadeh, H. (2011) An approach for solving fuzzy transportation problem. Applied Mathematical Sciences, 5(32), 1549-1566.

Hasan, M. K. (2012). Direct methods for finding optimal solution of a transportation problem are not always reliable. International Refereed Journal of Engineering and Science (IRJES), 1(2), 46-52.
المراجع

الجهاز المركزي للتعبئة العامة والإحصاء، الكتاب الإحصائي

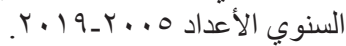

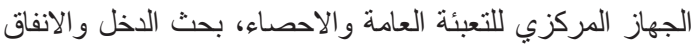

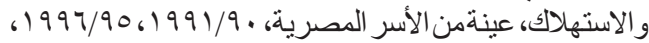


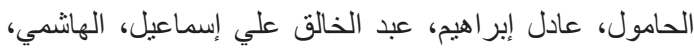

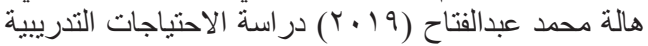

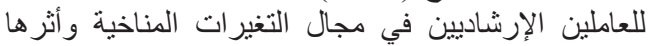

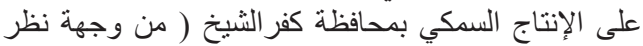

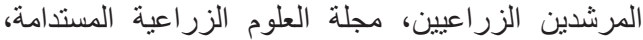

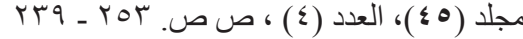

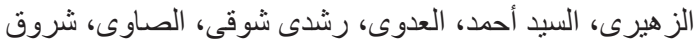

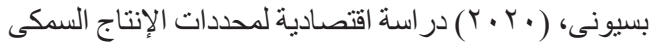

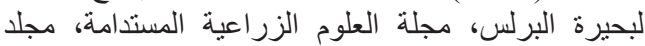

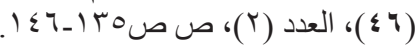

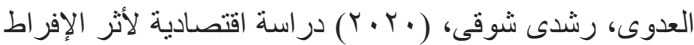

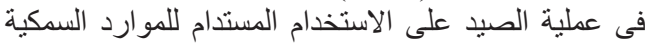
ببحيرة مريوط المصرية، مجلة العلوم الزراعية العبلة المستدامة،

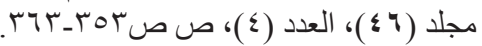

الملاحق

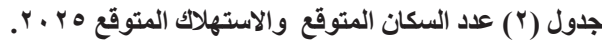

\begin{tabular}{|c|c|c|c|c|c|}
\hline الفرد كجم/ سنة استهلاك & عدد السكان المتوقع & معدل النمو & 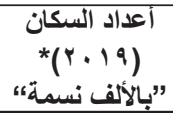 & 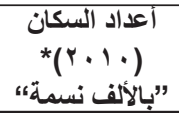 & المحافظة \\
\hline 9,9 & $T r \varepsilon r 0, \mu r$ &., $.5 \% 0$ & $q \vee \wedge \Lambda, V \varepsilon$ & VITV,IIA & القاهرة \\
\hline 0,1 & $1 \% \cdot|r, \Lambda|$ & $\therefore \mathrm{rVA}$ & $\wedge 910,11$ & $11 \cdot v, r 10$ & الجيزة \\
\hline 19,5 & $1 \leqslant r \wedge, V \Lambda$ &. .190 & $\Delta \Gamma 99, V \Gamma$ & $\{\mu T \Gamma, 1\rceil \Lambda$ & الاسكندرية \\
\hline$\sum 1, \Lambda$ & 971,91 &. $.5 \% 0$ & $V 1 \varepsilon, \Sigma 99$ & $1 \cdot \varepsilon,\{01$ & بورسعيد \\
\hline$r 1, r$ & $1 . \int T, \Gamma \varepsilon$ & 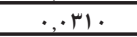 & $V \leqslant 9,10 V$ & $\Delta \leq q, \vee \Delta q$ & السيويب \\
\hline$\Gamma \varepsilon, 1$ & $\Gamma \cdots \Delta, v_{0}$ & $\therefore .170$ & $10 r 9 . \cdot 1$ & $111 \cdot .991$ & دمياط \\
\hline$I V, V$ & $\Lambda r \Delta 1,01$ &., $\int \Gamma \varepsilon$ & $17 \mathrm{Vq}, \mathrm{VV}$ & $\Delta r r \wedge, \Lambda r \mid$ & الدقطلية \\
\hline $1 \pi, 9$ & $900 \cdot, \cdot \varepsilon$ & $\therefore \Gamma 00$ & $V \varepsilon=1, V$ & $\Delta V Y T, 1 \leq \varepsilon$ & الشرقية \\
\hline$\Gamma, 9$ & $V r V \Lambda, V \Lambda$ & 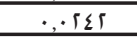 & OVQT,VV & $\varepsilon \Delta\{7, \Delta T \varepsilon$ & القليو بية \\
\hline$[1,1$ & $\varepsilon \mu \Gamma T, \Sigma \Lambda$ & $\therefore$. rIV & $r \leqslant V \Lambda, r v$ & 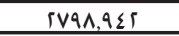 & كفر الثشبخ \\
\hline 11,1 & $\|\lceil\mid \varepsilon, 0$ &., $1 \wedge 9$ & $01 \leq 1, \sum 1$ & $\varepsilon\lceil T \Gamma, \Gamma$ & الغربية \\
\hline 5,1 & $\Delta 7\{\tau, 11$ & $\therefore$. 1 rq & $\varepsilon \Sigma \varepsilon I, v f$ & $r \leqslant 97, r \wedge$ & المنوفية \\
\hline 11,0 & $\Lambda \cdot \Lambda V, r \Lambda$ & $\therefore$. IRr & $7 \varepsilon \cdot \varepsilon, \Gamma 1$ & $0 . v 1, r \leq 1$ & البحيزة \\
\hline $11,$. & IVVV, 11 &..$\Gamma V^{\mu}$ & $1 T \Delta r, \Delta \Delta$ & $1 .\{9,1 \%$ & الاسماعيلية \\
\hline 5,9 & $\varepsilon r V O, V q$ &.$\cdot\lceil\Lambda \Lambda \uparrow$ & $r \Gamma \wedge \Lambda, \Gamma T$ & $\lceil\{V \cdot, q\rceil$ & بنى سويفت \\
\hline $1 \Gamma, 1$ & 0111,00 &.$\quad \mu r$. & $r V \leq V, q \varepsilon$ & rVII, $\leq \vee \wedge$ & الفيوم \\
\hline 0,5 & $V \mu T \varepsilon, 7 \varepsilon$ &.,$\cdot \Gamma \leqslant \Lambda$ & $\Delta V\{\Delta, \Gamma]$ & $\sum \sum \Lambda \Lambda 1, \Lambda \vee q$ & المنيا \\
\hline $0, r$ & $\Delta 1 \wedge \Delta, 9 \varepsilon$ & $\therefore .110$ & $\varepsilon \triangle \wedge V, \Delta \Lambda$ & $r v \cdot 1, r 99$ & أسيوط \\
\hline $0 . \varepsilon$ & ivrq, if &. .911 & 0194.0 & $\varepsilon \cdot \cdot, 1) \mu$ & سوهاج \\
\hline$v, r$ & $r r q \Lambda, \leqslant 9$ & $\therefore . .19$ & $r \mu \cdot \Gamma, \wedge q$ & $r \Gamma \cdot 9,9 \Lambda \Gamma$ & قن \\
\hline$\Lambda, V$ & $1 \wedge 10, r 0$ &., $.19 \mathrm{~V}$ & $10 r r, \varepsilon$ & $I \Gamma \Delta \Lambda, \Lambda \Lambda T$ & أسوان \\
\hline $1 \pi, 1$ & \&or, I1 &. .190 & rVI,ATI & $r \cdot 1, v[r$ & البحر الآحمر \\
\hline$\therefore$, vo & $r \| \Gamma \cdot \Lambda$ & $\therefore \Gamma \Gamma \varepsilon$ & {$[\leqslant 9.499$} & $199, \Gamma 1$ & الو ادي الجديد \\
\hline $9, \varepsilon$ & $1 \cdot 0,50$ & $\therefore . \cdot \mathrm{TV}]$ & $\Sigma 11, \Lambda \Sigma V$ & ror, rTo & مطروح \\
\hline $11, r$ & $\Delta \vee 0, \sum q$ &. .0110 & $\varepsilon 7 \pi, q \vee 0$ & $r v \varepsilon, \cdot v 1$ & شمال سيناء \\
\hline $1 \Lambda, V$ & $V r, \Sigma 0$ & $-\cdot, \cdot \mu \Lambda$. & $1 \cdot 0,90 r$ & $10 \leq, 9 \varepsilon 1$ & جنوب سيناء \\
\hline
\end{tabular}

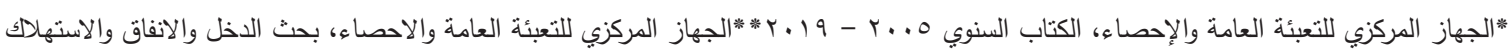

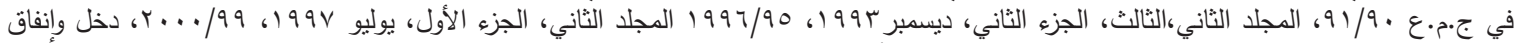

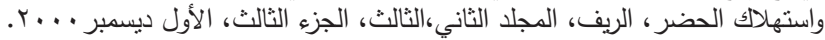




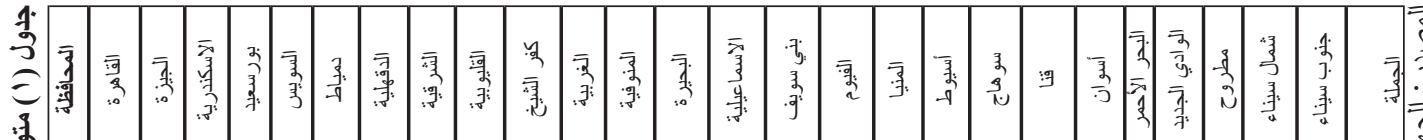

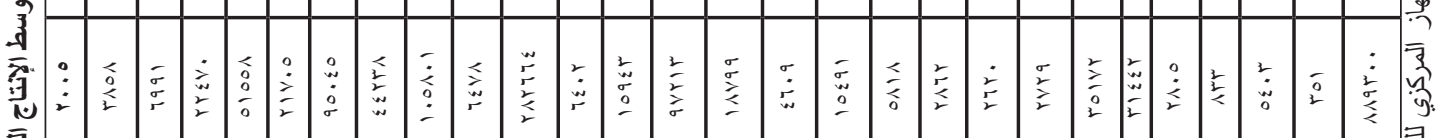

$\overline{3}$

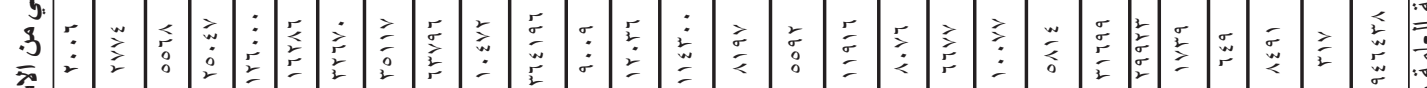

,

学

哥

司

\begin{tabular}{c}
0 \\
\hline \\
\hline
\end{tabular}

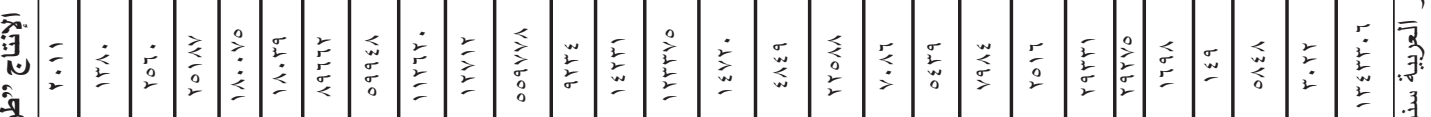

.

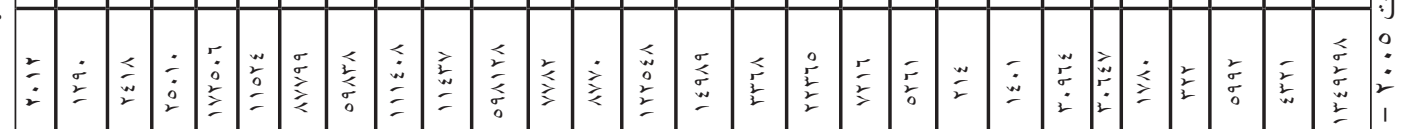

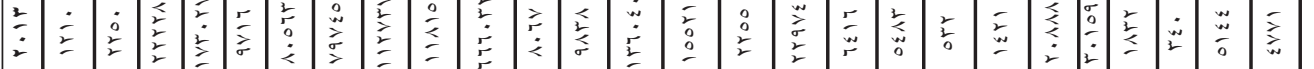

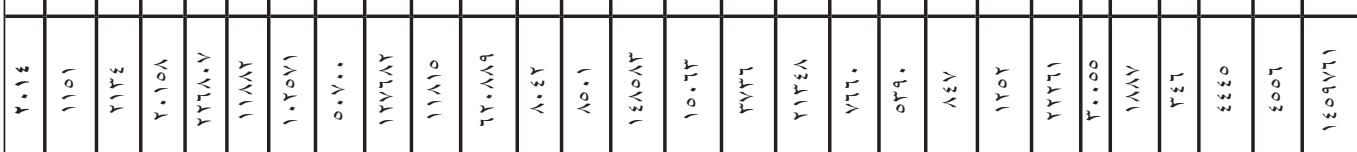

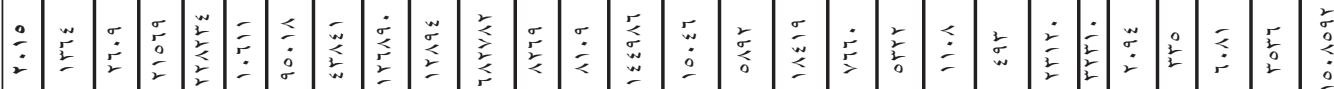

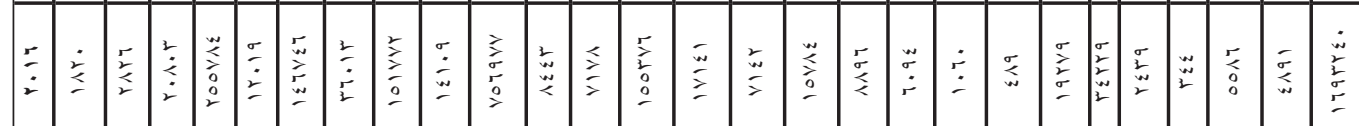

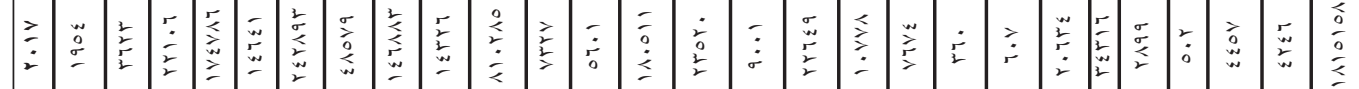

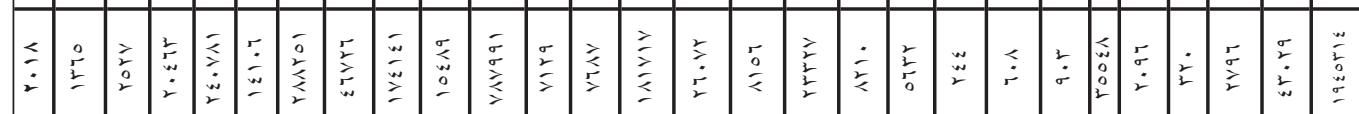

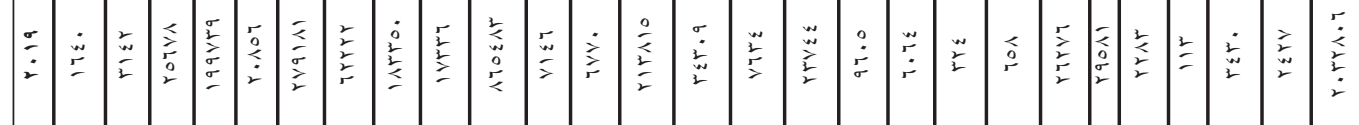

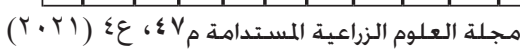




\title{
An Economic Study to Minimize the Costs of Transferring Surplus Local Fish Production between Governorates under Alternative Scenarios for the Expected Fish Production in 2025
}

\author{
Seham A. A. Hashim
}

Environmental Agric. Sci. Dept., College of Graduate Studies and Environmental Research, Ain Shams Univ., Cairo, Egypt

$\mathbf{T}$

HE STUDY aimed to identify the productive governorates with a production surplus and whose production exceeds their consumption, and the other governorates with a deficit whose consumption exceeds their production, and to determine the shortest marketing distances to raise the efficiency of the marketing process. The first scenario showed that the quantity expected to be transferred to the deficit governorates is about 632.5 thousand tons, representing $19.7 \%$ of the total production expected at the governorate level during the year 2025, and in light of this model, it is expected that The lowest cost of transportation is about 131.85 thousand monetary units $/ \mathrm{km} /$ thousand tons. The second pessimistic scenario showed, the average quantity expected to be transferred to the deficit governorates during 2025 is estimated at 715.84 thousand tons, representing about $30.7 \%$ of the total production of the governorates of the Republic during the year 2025, and the lowest cost of transportation is estimated at about 148.204 monetary units/km/thousand tons. The third optimistic scenario showed, the average quantity expected to be transferred to the deficit governorates is estimated at 536.835 thousand tons, representing about $12.32 \%$ of the average of the total governorates of the republic, and the lowest cost of transportation is estimated at 79.707 thousand monetary units $/ \mathrm{km} /$ thousand tons. The study recommended application of the proposed transport and distribution system between the governorates.

Keywords: Transportation model, Fish consumption, Local fish production, Average per capita fish production, Production deficit, Surplus production, population growth rate. 